\title{
Incidence of chronic atrophic gastritis: systematic review and meta-analysis of follow-up studies
}

\author{
Mariam Abdullahi Adamu - Melanie Nicole Weck • \\ Lei Gao $\cdot$ Hermann Brenner
}

Received: 5 August 2009/Accepted: 16 June 2010/Published online: 29 June 2010

(C) Springer Science+Business Media B.V. 2010

\begin{abstract}
Chronic atrophic gastritis (CAG) is an important precursor lesion of intestinal gastric cancer. As it is typically asymptomatic, epidemiological data on the incidence of CAG are sparse. We aimed to provide an overview of published data on CAG incidence (overall and according to risk factors) from follow-up studies. Articles with information on incidence of CAG published in English until 26th of July 2009 were identified through a systematic MEDLINE and EMBASE search. Data extracted include study characteristics and key findings regarding the incidence of CAG. A meta-analysis was performed on the association between Helicobacter pylori infection and CAG incidence. Overall, data on CAG incidence were available from 14 studies, in 7 studies incidence could be estimated according to $H$. pylori infection. Most studies were conducted in symptomatic or high risk populations and the maximum number of incident cases was 284 . Incidence estimates ranged from 0 to $11 \%$ per year and were consistently below $1 \%$ in patients not infected with H. pylori. The highest incidence was observed in a special study conducted on ulcer patients treated by proximal gastric vagotomy. Rate ratios for the association between H. pylori infection and CAG incidence ranged from 2.4 to 7.6 with a summary estimate of 5.0 (95\% confidence
\end{abstract}

Electronic supplementary material The online version of this article (doi:10.1007/s10654-010-9482-0) contains supplementary material, which is available to authorized users.

M. A. Adamu · M. N. Weck $\cdot$ L. Gao $\cdot$ H. Brenner $(\bowtie)$ Division of Clinical Epidemiology and Aging Research, German Cancer Research Center, Bergheimer Str. 20, 69115 Heidelberg, Germany

e-mail: h.brenner@dkfz-heidelberg.de

M. A. Adamu

e-mail: m.adamu@dkfz-heidelberg.de interval: $3.1-8.3$ ). Incidence of CAG is very low in the absence of $H$. pylori infection. There is a need for more population-based studies to provide comparable estimates of incidence and the impact of risk factors in the development of CAG.

Keywords Chronic atrophic gastritis - Incidence · Follow-up studies
Abbreviations
CAG Chronic atrophic gastritis
cagA Cytotoxin associated gene A
H. pylori Helicobacter pylori
PG Pepsinogen

\section{Introduction}

Chronic atrophic gastritis (CAG) is an important precursor lesion in the development of intestinal type of gastric cancer [1]. Even though there is a worldwide decline in the incidence of this type of cancer, its public health burden is still high, with gastric cancer ranking fourth in cancer incidence and being the second most common cause of cancer-related deaths worldwide [2].

The sequence of events that occur in the gastric mucosa before the manifestation of gastric cancer has been shown to take decades. Typically, the sequence is from chronic non-atrophic gastritis that progresses to $\mathrm{CAG}$, which may eventually lead to intestinal metaplasia, dysplasia and finally adenocarcinoma [3]. In order to understand the processes involved in the occurrence of this type of cancer and to establish risk factors for its development, it is 
necessary to understand its precursor lesions and the risk factors associated with their development.

The first follow-up studies on CAG have been conducted more than five decades ago. However, the majority of epidemiological studies on CAG and its risk factors have been cross-sectional [4]. Infection with the gastric bacterium Helicobacter pylori was shown to be strongly associated with $\mathrm{CAG}$ in a recently published meta-analysis that relied on cross-sectional data [5]. However, to prove the causal role of $H$. pylori infection and other risk factors in the development of $\mathrm{CAG}$, analysis of follow-up data is necessary.

The aim of this systematic review is to provide an overview of the incidence of CAG, both overall and according to potential risk factors reported by follow-up epidemiological studies.

\section{Materials and methods}

Articles providing information on the incidence of $\mathrm{CAG}$ were identified through a systematic search in the MEDLINE and EMBASE databases by using various combinations of the terms 'atrophic', 'atrophy', 'chronic', 'gastritis', 'pepsinogen', 'incidence', 'cohort', 'follow-up', 'long term', 'prospective' and 'retrospective'. Additionally, references of retrieved articles were screened for further studies (cross-referencing).

All original research articles from human studies published in English until the 26th of July 2009 were included. Due to the few studies that could be identified, restrictions in terms of selection criteria of participants (e.g., hospital based studies or studies which included patients with a particular disease or treatment) were not taken. However, only studies with more than 15 study participants without CAG at baseline and with a mean or median of at least 2 years of follow-up were included. Furthermore, studies in which follow-up was made by indication only (rather than by design) were not included.

The following data were extracted from the articles: first author, year of publication, characteristics of the study population [country, population type, sample size, age (range and mean or median) of study participants], dates of baseline and of follow-up examinations, mean years of follow-up, $H$. pylori infection at baseline, method of diagnosis of CAG (both at baseline and at follow-up), absolute number of incident cases and incidence of CAG (in percent per year), overall and according to pertinent risk factors. If incidence rates of CAG per year were not given in the articles, they were calculated from case numbers and mean years of follow-up time. In order to enhance comparability of the studies only data from the corpus or fundus mucosa (where given separately) were used to calculate incidence rates in the studies with histological assessment of CAG even when data on multiple biopsy sites for histological assessment of CAG was provided. Extraction of data from the articles was performed independently by two investigators (MAA and LG), and minor discrepancies were resolved in consensus.

A meta-analysis on the association between $H$. pylori infection and incidence of CAG was conducted with the Comprehensive Meta-Analysis software (V2.0; Biostat, Englewood, NJ, USA) by using a random effects model. The meta-analysis was performed on all studies where incidence of CAG could be estimated according to H. pylori infection at baseline. A forest plot showing rate ratios for the incidence among $H$. pylori positive compared to $H$. pylori negative study participants was created. Heterogeneity was analysed by the $Q$-test [6] and by the $I^{2}$-test [7]. Publication bias was analysed by Egger's weighted regression model [8] and by Begg's rank correlation method [9].

\section{Results}

Figure 1 presents a flowchart of the literature search; numbers of articles and reasons for exclusion are given. The literature search initially resulted in 3,334 articles which were screened by title and abstract. Sixty-four articles were identified to potentially provide data on the incidence of CAG. After reading the full text of the 64 articles a number of them had to be excluded, these are given in an additional file as supplementary material. 8 articles were excluded because they did not present any data on CAG or its incidence. 15 articles had to be excluded because they only presented prevalence data at baseline and follow-up examinations or at either time period. In another 14 articles, distribution or changes in

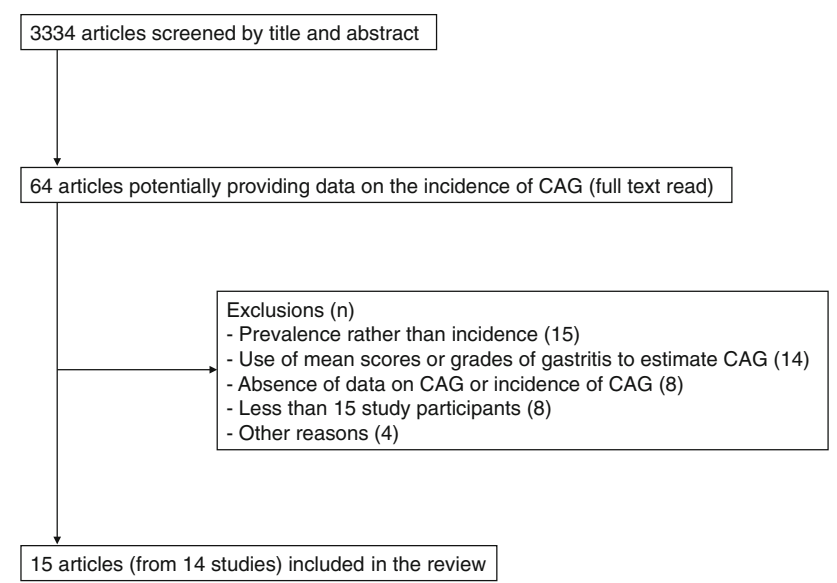

Fig. 1 Flow chart representing the literature search. Abbreviations: $C A G$ chronic atrophic gastritis 
severity of carditis or gastritis scores, or gastritis grades rather than incidence of CAG was reported. 8 articles were excluded because they had less than 15 study participants at baseline without CAG. Another 4 articles were excluded for the following reasons: all patients had either chronic gastritis or CAG at baseline, incidence of CAG was based on biopsies and not on study participants, incidence of CAG and metaplasia or either of them were grouped together and in one study, no mean follow-up time could be estimated. Due to these exclusions, only 14 studies (from 15 articles) with information on the incidence of CAG could be included in this review [10-24]. According to the above described criteria, all of these studies included more than 15 study participants who were diagnosed to be free of CAG at baseline, either by histology ('normal' or 'superficial gastritis') or by serum pepsinogen (PG) levels.

A summary and characterization of the 14 studies included in this review is given in Table 1. The earliest study was started in 1952 in Finland [15]. The most recent one in 1990-1993 in Slovenia; [19] all studies were published between 1988 and 2008. 2 of the studies were conducted in multiple centres in different countries [20,22]. Studies were carried out in Sweden, [10] Columbia, [11] the Netherlands, [12,13] the Netherlands and Sweden, [14] Finland, [15] Estonia, [16, 24] Japan, [17, 23] USA, [18] Slovenia, [19] Australia, Canada, Germany, and the Netherlands, [20] Germany [21] and Denmark, Finland, Norway and Sweden [22]. Most of the studies were not population-based and consisted of symptomatic patients. CAG was diagnosed by histology in twelve studies and by serum pepsinogen levels in two studies [17, 23]. Mean follow-up time of the study participants in the different studies ranged from 2 to 32 years. Sample sizes ranged from 17 to a maximum of 780 subjects. However, only 6 of the studies had more than 100 participants and only one study included more than 500 study participants. Numbers of incident cases ranged from 0 to 284 . The age of the study participants at baseline ranged from 15 to at least 83 or older. Where reported, mean age at baseline ranged from 35 to 64 years.

12 studies examined $H$. pylori infection at baseline by one or more of the following methods: bacterial culture, [12, 13, 19] histology, [12-16, 18-22, 24] PCR (polymerase chain reaction), [19] rapid urease test, [19] and serology [12-14, 17, 19, 24].

Incidence rates of CAG ranged from 0 to $10.9 \%$ per year. The lowest incidence $(0 \%)$ was observed in two study populations, one made up of a very special cohort of 71 patients with reflux esophagitis, who were treated by fundoplication (an endoscopic procedure of wrapping the fundus around the esophagus for treatment of reflux esophagitis) and followed for a mean period of 5 years [14]. The other study population consisted of 45 patients who had undergone successful $H$. pylori eradication therapy before the start of the study [19]. However, there was also a lack of any incident case in subpopulations of 140 $H$. pylori negative patients who had undergone either fundoplication or who were treated by omeprazole for 7 years and were followed for 7 years [22]. The highest incidence was estimated in a cohort of patients who had undergone proximal gastric vagotomy for treatment of juxtapyloric ulcer [10].

The two studies from Japan which diagnosed CAG by serum pepsinogen levels, a study from Estonia [24] and one from Colombia [11] were the only studies with an approximate population-based study sample. The incidence observed in the study from Estonia was 1.9\% per year and that in the Columbian study $7.1 \%$. The two Japanese studies used slightly different definitions of CAG. The incidence reported in the first of these studies using a less restrictive CAG definition (PGI $<70 \mathrm{ng} / \mathrm{mL} \&$ PGI/ PGII $<3)$ was rather high $(2.7 \%$ per year $)$ [17] compared to the studies that assessed CAG by histology. The incidence in the second serology-based study was $1.3 \%$ per year [23].

In the study from Columbia [11], incidence of CAG was given according to age groups with a higher incidence in the older group $(\leq 40$ years of age $7.0 \%$ per year and $\geq 41$ years of age $8.5 \%$ per year). There was however, no such trend in another two studies in which incidence of CAG could be estimated by the given age groups [17, 23]. In two serological studies from Japan incidence was further estimated by gender, with males (3.2\% per year) having higher CAG incidence compared to females $(2.4 \%$ per year) in one of the studies [17], whereas no such difference was seen in the other one (annual incidence in males and females: 1.3 and $1.4 \%$, respectively) [23]. The study of Ozasa et al. [17] from Japan was the only study in which incidence of CAG could be estimated by dietary and lifestyle factors. Daily consumption of green-yellow vegetables was associated with an increased incidence of CAG in both men and women. Overall, daily alcohol consumption was also associated with an increased incidence of CAG, however, results were not consistent across genders. Men who smoked daily had a higher incidence of CAG compared to those who did not smoke daily; the reverse was the case in the female study participants. Crude incidence of CAG according to these risk factors (daily smoking, daily alcohol consumption and daily consumption of green yellow-vegetables) is given as footnotes in Table 1.

In 7 studies, incidence rates could be estimated according to $H$. pylori infection at baseline. In all of these studies, incidence rates were substantially higher in $H$. pylori positive subjects (up to $8.1 \%$ per year) compared to the $H$. pylori negatives (up to $1.8 \%$ per year). Of the studies that assessed $H$. pylori infection, only one provided 


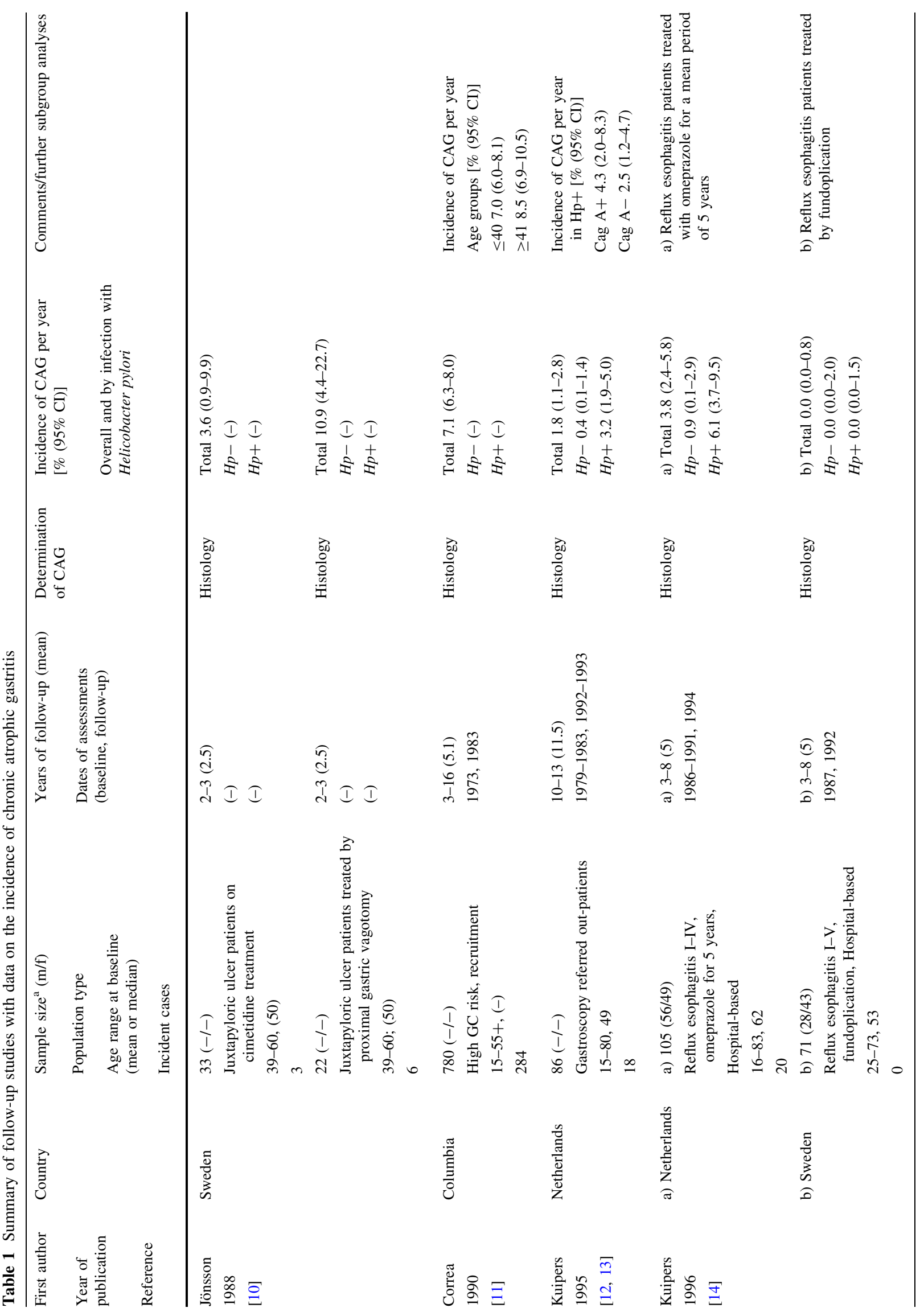




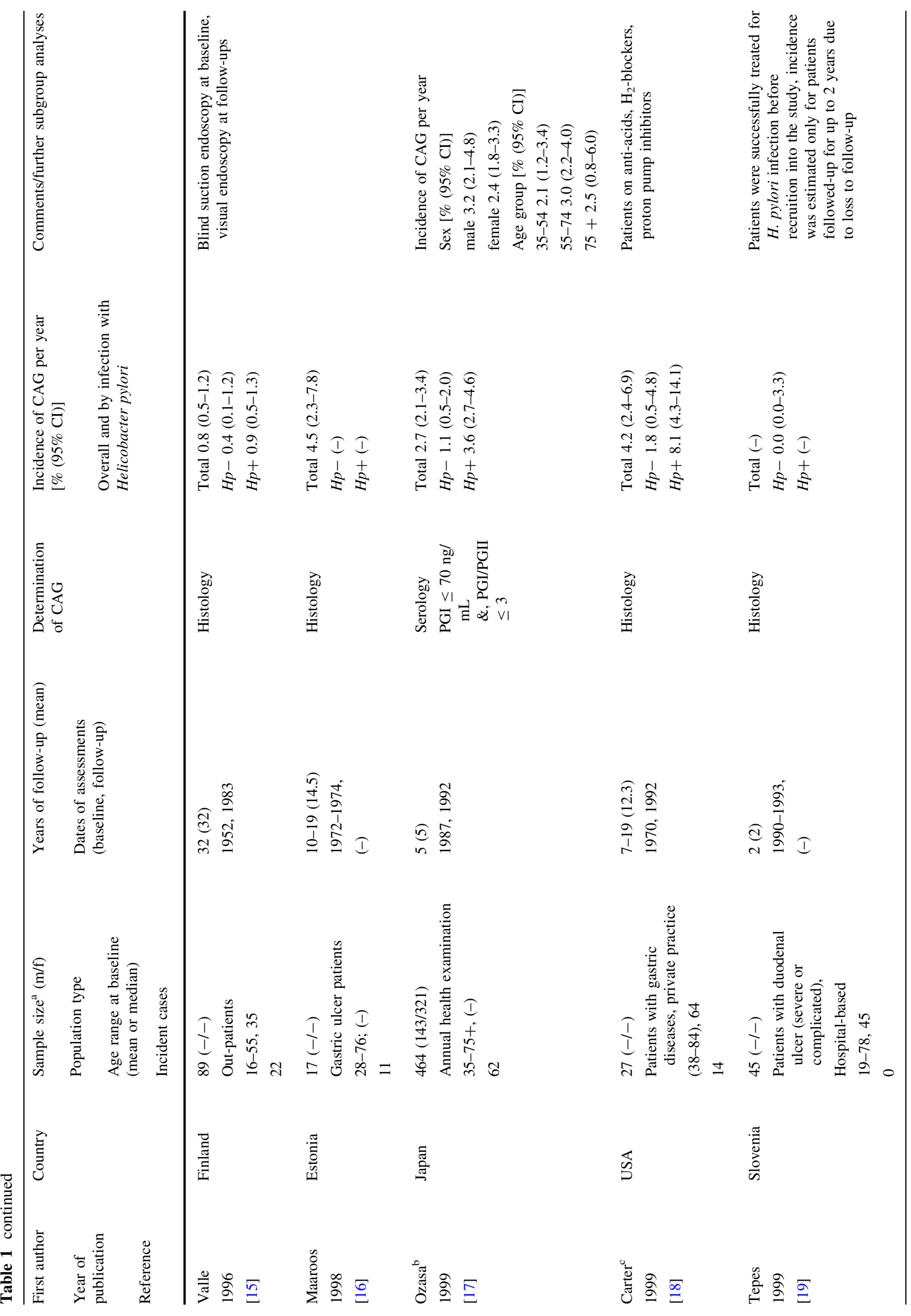




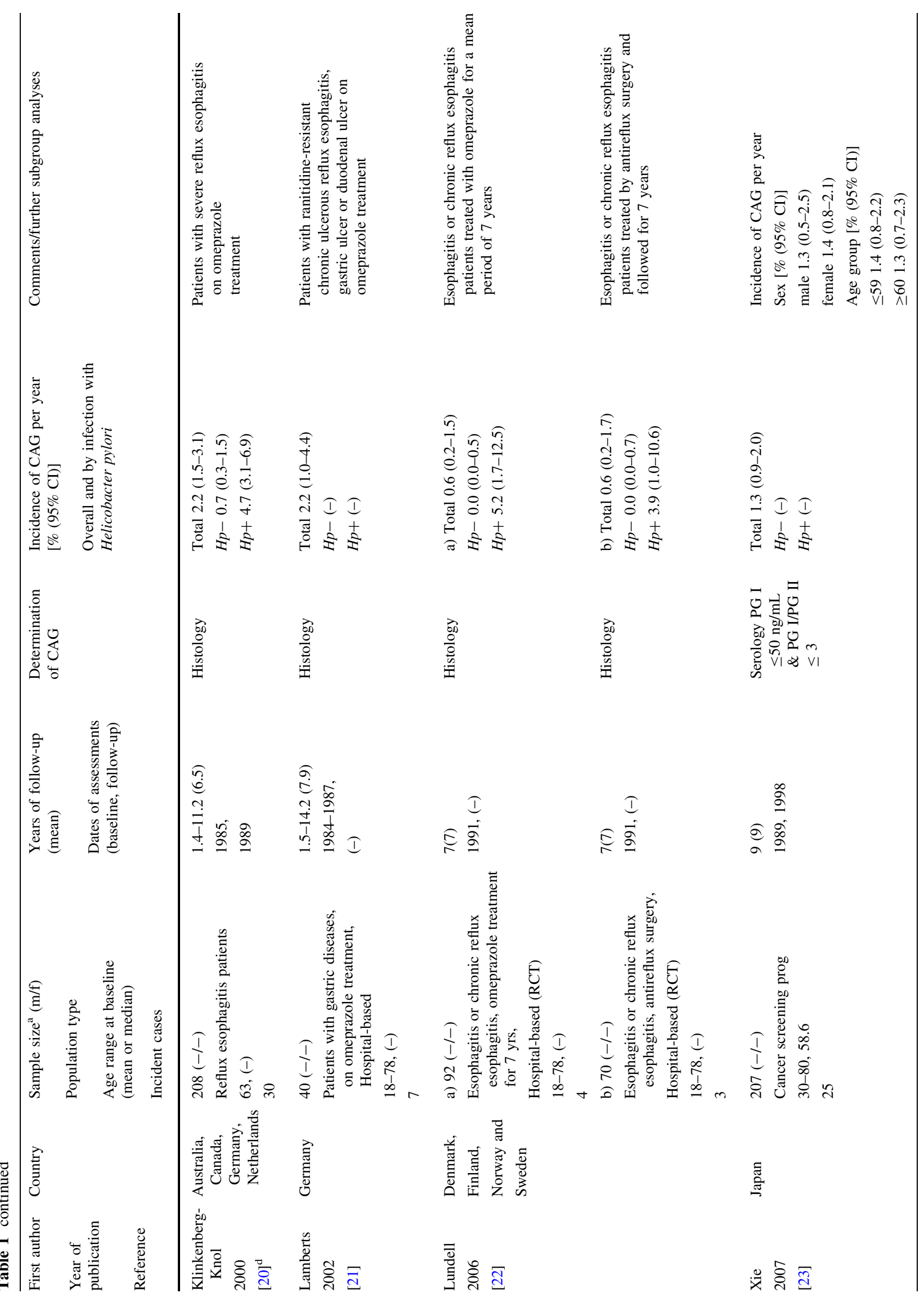




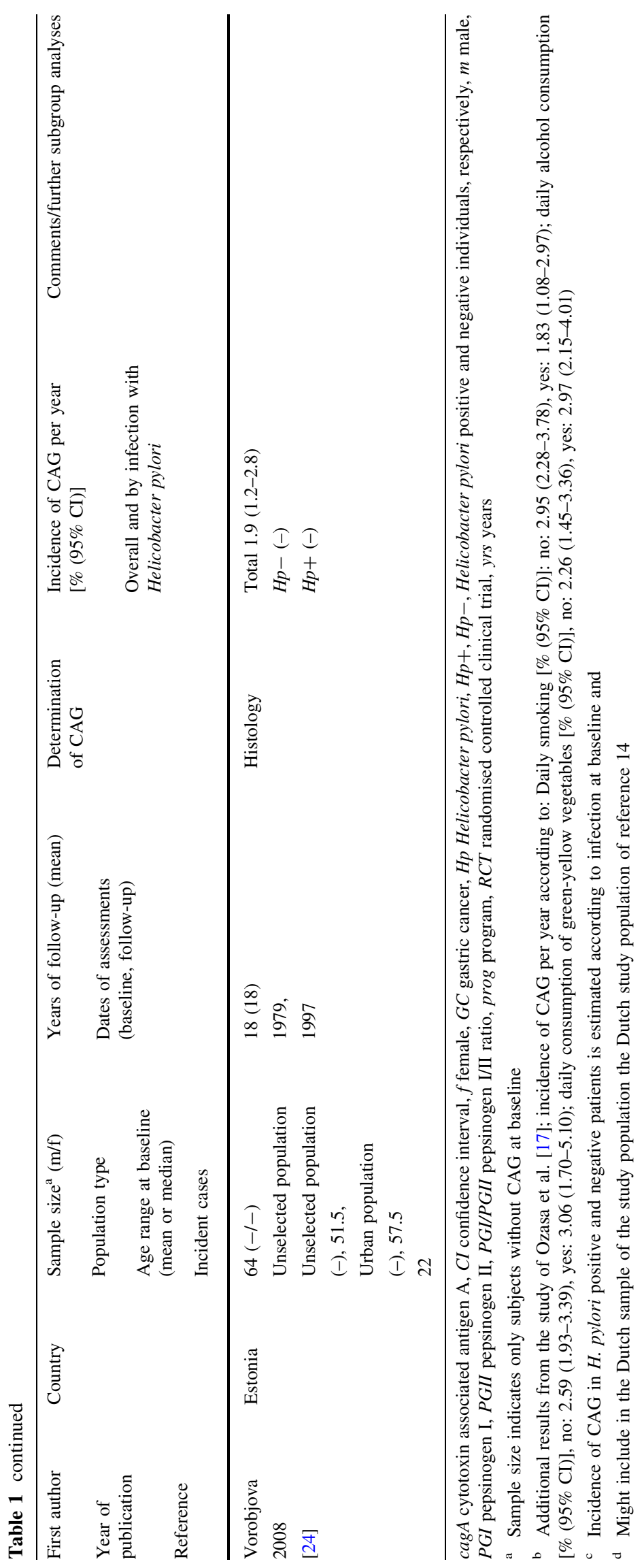


Fig. 2 Meta-analysis on the association between $H$. pylori and incidence of chronic atrophic gastritis (CAG). * Incident CAG-cases per personyears among the group of $H$. pylori positive and $H$. pylori negative study participants, respectively. Abbreviation: $C I$ confidence interval, $R R$ rate ratio

$\begin{array}{lllll}\text { Study } & \text { RR } & \mathbf{9 5 \%} \mathbf{C l} & \text { H. pylori +* } & \text { H. pylori - * } \\ \text { Valle 1996 } & 2.4 & 0.6-10.1 & 20 / 2304 & 2 / 544 \\ \text { Kuipers 1995 } & 7.6 & 1.8-33.2 & 16 / 506 & 2 / 483 \\ \text { Kuipers 1996 } & 6.8 & 1.6-29.5 & 18 / 500 & 2 / 380 \\ \text { Carter 1999 } & 4.6 & 1.3-16.4 & 11 / 136 & 3 / 170 \\ \text { Ozasa 1999 } & 3.3 & 1.6-6.6 & 53 / 1490 & 9 / 830 \\ \text { Klinkenberg-Knol 2000 } & 6.7 & 2.7-16.3 & 24 / 507 & 6 / 845 \\ \text { Lundell 2006 } & 95.5 & 5.5-1671.3 & 7 / 154 & 0 / 980 \\ & & & & \\ \text { Summary estimate } & \mathbf{5 . 0} & \mathbf{3 . 1 - 8 . 3} & & \end{array}$

Rate ratio and $95 \% \mathrm{Cl}$

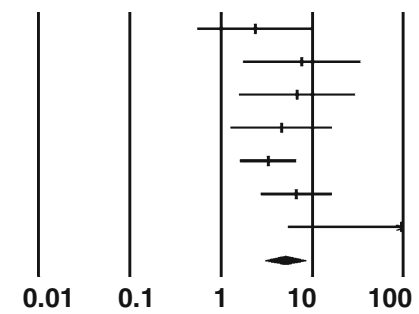

additional data on antibodies against cytotoxin associated gene A (cagA), an established virulence factor of $H$. pylori. Among the H. pylori positive population in this study, incidence was substantially higher in subjects who showed antibodies against cagA ( $4.3 \%$ per year) compared to those who were cagA-negative (2.5\% per year) [12].

Estimates of the impact of $H$. pylori infection on the incidence of CAG are shown in Fig. 2. The forest plot visualizes results of a meta-analysis conducted among all those studies which provided information on $H$. pylori infection of the study participants at baseline. The test for heterogeneity resulted in $I^{2}=18.4(Q$-test: $P>0.2)$ and there was no indication of publication bias according to both Egger's weighted regression model and Begg's rank correlation method $(P>0.1)$. Rate ratios comparing the incidence of CAG in $H$. pylori positive subjects to the incidence in $H$. pylori negative subjects ranged from 2.4 to 7.6 (with an outlier at 95.5). In a sensitivity analysis excluding the study with a rate ratio of 95.5 [22], the summary estimate was only slightly lower $[4.5,95 \%$ confidence interval (CI): 2.9-7.0]. We furthermore, performed a meta-analysis including only study populations made up of patients with gastric complains or diseases in which CAG was diagnosed exclusively by gastroscopy. In this analysis, heterogeneity was further reduced $\left[I^{2}=9.5(Q-\right.$ test: $P>0.4)]$ and the summary estimate $[6.195 \% \mathrm{CI}$ (3.4-10.9)] was similar to the one obtained when all the studies were included.

\section{Discussion}

Overall, fourteen studies with data on the incidence of CAG could be identified. Among these, incidence rates varied widely, ranging from 0 to $10.9 \%$ per year. Substantially higher incidence rates were consistently observed in $H$. pylori positive participants compared to $H$. pylori negatives. The meta-analysis on the association between H. pylori infection and incidence of CAG resulted in a summary estimate of 5.0 (95\% confidence interval: 3.1-8.3). Patients who had undergone proximal gastric vagotomy, as well as patients who have reflux esophagitis and who were on long-term omeprazole treatment showed a remarkable high incidence of CAG.

The much higher incidence rates of CAG that were observed in $H$. pylori positive individuals compared to $H$. pylori negative individuals and the according rate ratios observed in the meta-analysis are consistent with findings from a number of cross-sectional studies which disclosed a strong association between $H$. pylori infection and CAG [5]. Similarly, the $H$. pylori specific virulence factor cagA has consistently been shown to be associated with an increased risk of CAG in cross-sectional studies. In this review, only one of the studies took cagA seropositivity into account and within the group of $H$. pylori positive individuals, those who did not show antibodies against cagA showed a much lower incidence than those who were cagA-positive [12]. Even though these results have to be interpreted with caution due to the small sample size of the study, they support the importance and impact of cagA in the pathogenesis of $H$. pylori induced CAG.

The highest incidence of CAG was observed in a cohort of patients treated by vagotomy for juxtapyloric ulcer [10]. High incidence rates of CAG were furthermore, observed in cohorts of $H$. pylori positive patients treated with omeprazole for reflux esophagitis [14, 20, 22]. This finding might be explained by the joint impact of long-term $H$. pylori infection and omeprazole therapy on lowering of the gastric acidity which makes the gastric mucosa more prone to atrophy. Furthermore, even though, the overall incidence of CAG in this group of patients was remarkably high, CAG incidence was lower and comparable to that of other study populations without such treatment in the H. pylori negative study participants, leading us to presuppose that infection with the bacterium plays a major role for the development of CAG in this group of patients.

Even though $H$. pylori can survive the acidic gastric environment, due to its ability to break down urea to carbon dioxide and ammonia by urease activity, it has been shown to have an optimal growth at neutral $\mathrm{pH}[25,26]$. In H. pylori positive patients, treatment with omeprazole or proximal gastric vagotomy reduces the gastric acidity and might, enhance bacterial growth. Bacterial overgrowth might be a key factor in the development of atrophy in such 
patients and therefore, maybe this higher incidence of CAG seen in this group of patients. Therefore, eradication of H. pylori infection, which has been shown to be able to reverse gastritis without exacerbation of reflux disease, maybe particularly important in this group of patients [27].

A number of limitations of this systematic review have to be kept in mind. Only studies published in English as full articles were included. Possibly, additional results have been presented at conferences and published as meeting proceedings. However, very few follow-up studies on the incidence of CAG have been conducted so far and it seems rather unlikely that further relevant studies exclusively reported in abstract form exist from which appropriate details on incidence could be derived.

Several other aspects have to also be considered when interpreting the study results. The determination of CAG requires specific attention. Even though, almost all of the studies included in this review assessed CAG by histological assessment of biopsy specimen of the gastric mucosa, which is considered the gold standard for clinical diagnoses of CAG, it should be noted that incidence of CAG was estimated solely on the corpus or fundus mucosa and did not take into account the antral mucosa (where data were separately given for each). It has been suggested, that the antral mucosa might be more prone to regressive $\mathrm{CAG}$ changes and that atrophy of the body mucosa might be associated with healing of the antrum [28]. On the other hand, atrophy has been reported to start in the antrum and to spread proximally (thus leading to an increased incidence in the antrum compared to the corpus) [29]. In the two Japanese studies, different cut points of serum PG levels were used to define CAG [17, 23]. This difference might explain the variation of the reported incidence by a factor of more than 2 (lower incidence in the study with the more stringent definition of disease), despite the similarity in setting, age range and recruitment years of the study populations. These findings underline the importance of the choice of the cut-off level [30, 31].

The main limitations regarding existing evidence are related to the heterogeneity of the existing studies. For example, the use of different diagnostic methods for assessment of CAG hampers direct comparisons between the studies. Similarly, H. pylori infection was assessed by different methods. As sensitivity and specificity of the different tests used to determine infection vary, misclassification of infection status might occur, leading to underestimation of the impact of $H$. pylori on the development of CAG. Most of the studies that could be included in the review showed rather small sample sizes. Additionally, only one of the study populations was sampled from the general population and most participants were lost to follow-up. Some studies had a rather short follow-up time and information on incidence according to potential risk factors other than $H$. pylori was lacking almost entirely.

Despite these limitations, there is consistent evidence that incidence of CAG is very low in the absence of H. pylori infection. These findings are consistent with a major role of $H$. pylori infection suggested previously by cross-sectional studies. Proximal gastric vagotomy and long-term treatment with omeprazole of $H$. pylori positive patients with reflux esophagitis might further increase the risk to develop $\mathrm{CAG}$, requiring eradication of the infection. However, this review also clearly shows the lack of largescale and population-based studies on the incidence of CAG. Ideally, studies could be designed in such a way that they allow for assessment of international variation as well as temporal trends. Using non-invasive tests (such as serum PG concentrations) [32] for the diagnosis of CAG might greatly enhance possibilities to conduct such studies in population-based (asymptomatic) study samples. In order to further clarify the role of age, gender, socio-demographic factors, diet and lifestyle factors, such as smoking on the incidence of CAG future follow-up studies should address these, as well as the role of any other potential risk factors.

Acknowledgements This work was supported by a grant from the Baden-Württemberg State Ministry of Research, Science and Arts. The work of Gao Lei was supported by a scholarship from the German Academic Exchange Service.

\section{References}

1. Ohata H, Kitauchi S, Yoshimura N, Mugitani K, Iwane M, Nakamura H, Yoshikawa A, Yanaoka K, Arii K, Tamai H, Shimizu Y, Takeshita T, Mohara O, Ichinose M. Progression of chronic atrophic gastritis associated with Helicobacter pylori infection increases risk of gastric cancer. Int J Cancer. 2004;109:138-43.

2. Parkin DM, Bray F, Ferlay J, Pisani P. Global cancer statistics, 2002. CA Cancer J Clin. 2005;55:74-108.

3. Kuipers EJ. Review article: exploring the link between Helicobacter pylori and gastric cancer. Aliment Pharmacol Ther. 1999; 13(Suppl 1):3-11.

4. Weck MN, Brenner H. Prevalence of chronic atrophic gastritis in different parts of the world. Cancer Epidemiol Biomarkers Prev. 2006;15:1083-94.

5. Weck MN, Brenner H. Association of Helicobacter pylori infection with chronic atrophic gastritis: meta-analyses according to type of disease definition. Int J Cancer. 2008;123:874-81.

6. Cochran WG. The combination of estimates from different experiments. Biometrics. 1954;10:101-29.

7. Higgins JP, Thompson SG, Deeks JJ, Altman DG. Measuring inconsistency in meta-analyses. BMJ. 2003;327:557-60.

8. Egger M, Davey Smith G, Schneider M, Minder C. Bias in metaanalysis detected by a simple, graphical test. BMJ. 1997;315: 629-34.

9. Begg CB, Mazumdar M. Operating characteristics of a rank correlation test for publication bias. Biometrics. 1994;50:1088-101. 
10. Jonsson KA, Strom M, Bodemar G, Norrby K. Histologic changes in the gastroduodenal mucosa after long-term medical treatment with cimetidine or parietal cell vagotomy in patients with juxtapyloric ulcer disease. Scand J Gastroenterol. 1988;23:433-41.

11. Correa P, Haenszel W, Cuello C, Zavala D, Fontham E, Zarama G, Tannenbaum S, Collazos T, Ruiz B. Gastric precancerous process in a high risk population: cohort follow-up. Cancer Res. 1990;50:4737-40.

12. Kuipers EJ, Perez-Perez GI, Meuwissen SG, Blaser MJ. Helicobacter pylori and atrophic gastritis: importance of the cagA status. J Natl Cancer Inst. 1995;87:1777-80.

13. Kuipers EJ, Uyterlinde AM, Pena AS, Roosendaal R, Pals G, Nelis GF, Festen HPM, Meuwissen SGM. Long-term sequelae of Helicobacter pylori gastritis. Lancet. 1995;345:1525-8.

14. Kuipers EJ, Lundell L, Klinkenberg-Knol EC, Havu N, Festen HP, Liedman B, Lamers CB, Jansen JB, Dalenback J, Snel P, Nelis GF, Meuwissen SG. Atrophic gastritis and Helicobacter pylori infection in patients with reflux esophagitis treated with omeprazole or fundoplication. N Engl J Med. 1996;334:1018-22.

15. Valle J, Kekki M, Sipponen P, Ihamaki T, Siurala M. Long-term course and consequences of Helicobacter pylori gastritis. Results of a 32-year follow-up study. Scand J Gastroenterol. 1996;31: 546-50.

16. Maaroos HI, Havu N, Sipponen P. Follow-up of Helicobacter pylori positive gastritis and argyrophil cells pattern during the natural course of gastric ulcer. Helicobacter. 1998;3:39-44.

17. Ozasa K, Kurata JH, Higashi A, Hayashi K, Inokuchi H, Miki K, Tada M, Kawai K, Watanabe Y. Helicobacter pylori infection and atrophic gastritis: a nested case-control study in a rural town in Japan. Dig Dis Sci. 1999;44:253-6.

18. Carter M, Katz DL, Haque S, DeLuca VA. Jr. Does acid suppression by antacids and $\mathrm{H} 2$ receptor antagonists increase the incidence of atrophic gastritis in patients with or without $H$. pylori gastritis? J Clin Gastroenterol. 1999;29:183-7.

19. Tepes B, Kavcic B, Zaletel LK, Gubina M, Ihan A, Poljak M, Krizman I. Two- to four-year histological follow-up of gastric mucosa after Helicobacter pylori eradication. J Pathol. 1999;188: 24-9.

20. Klinkenberg-Knol EC, Nelis F, Dent J, Snel P, Mitchell B, Prichard P, Lloyd D, Havu N, Frame MH, Roman J, Walan A. Long-term omeprazole treatment in resistant gastroesophageal reflux disease: efficacy, safety, and influence on gastric mucosa. Gastroenterology. 2000;118:661-9.

21. Lamberts R, Brunner G, Solcia E. Effects of very long (up to 10 years) proton pump blockade on human gastric mucosa. Digestion. 2001;64:205-13.
22. Lundell L, Havu N, Miettinen P, Myrvold HE, Wallin L, Julkunen R, Levander K, Hatlebakk JG, Liedman B, Lamm M, Malm A, Walan A. Changes of gastric mucosal architecture during long-term omeprazole therapy: results of a randomized clinical trial. Aliment Pharmacol Ther. 2006;23:639-47.

23. Xie XF, Ito M, Yoshihara M, Haruma K, Tanaka S, Chayama K. Serum pepsinogen levels in the Japanese population: prospective study of 9 years of follow-up. Hepatogastroenterology. 2007;54: 1887-90.

24. Vorobjova T, Maaroos HI, Uibo R. Immune response to Helicobacter pylori and its association with the dynamics of chronic gastritis in the antrum and corpus. APMIS. 2008;116:465-76.

25. Sidebotham RL, Worku ML, Karim QN, Dhir NK, Baron JH. How Helicobacter pylori urease may affect external $\mathrm{pH}$ and influence growth and motility in the mucus environment: evidence from in vitro studies. Eur J Gastroenterol Hepatol. 2003;15: 395-401.

26. Burne RA, Chen YY. Bacterial ureases in infectious diseases. Microbes Infect. 2000;2:533-42.

27. Kuipers EJ, Nelis GF, Klinkenberg-Knol EC, Snel P, Goldfain D, Kolkman JJ, Festen HP, Dent J, Zeitoun P, Havu N, Lamm M, Walan A. Cure of Helicobacter pylori infection in patients with reflux oesophagitis treated with long term omeprazole reverses gastritis without exacerbation of reflux disease: results of a randomised controlled trial. Gut. 2004;53:12-20.

28. Ihamaki T, Kekki M, Sipponen P, Siurala M. The sequelae and course of chronic gastritis during a 30- to 34-year bioptic followup study. Scand J Gastroenterol. 1985;20:485-91.

29. El-Zimaity HM, Ota H, Graham DY, Akamatsu T, Katsuyama T. Patterns of gastric atrophy in intestinal type gastric carcinoma. Cancer. 2002;94:1428-36.

30. Weck MN, Stegmaier C, Rothenbacher D, Brenner H. Epidemiology of chronic atrophic gastritis: population-based study among 9444 older adults from Germany. Aliment Pharmacol Ther. 2007;26:879-87.

31. Brenner H, Rothenbacher D, Weck MN. Epidemiologic findings on serologically defined chronic atrophic gastritis strongly depend on the choice of the cutoff-value. Int J Cancer. 2007;121: 2782-6.

32. Iijima K, Abe Y, Kikuchi R, Koike T, Ohara S, Sipponen P, Shimosegawa T. Serum biomarker tests are useful in delineating between patients with gastric atrophy and normal, healthy stomach. World J Gastroenterol. 2009;15:853-9. 